\title{
Dualität bei Steuerungsproblemen mehrfacher Integrale
}

\author{
S. Pickenhatn
}

Es werden Dualitätsbeziehungen zu Steuerúngsproblemen mehrfacher Integrale untersucht. Im Mittelpunkt steht die Frage nach starker Dualitït. Durch nochmalige Dualisierung der dualen Aufgabe gelingt es, Beziehungen zwischen Steuerungsproblemen und Flußproblemen im Sinne von Young und KLöTZLER herzuleiten und einen möglichen neuen Zugang zu Aussagen . über starke Dualität bei mehrdimensionalen Problemen zu finden.

Исследуются - соотношения двоиственности для задач оптимального управления с кратным̈и интегралами. В центре пнтереса стоит вопрос. о сильной двоп̆ствениости. С помощью рассмотрения бидуальной задачи удается найти соотношения между проблемами оптимального управления и потоковыми проблемами в смысле ЯнгА и КлёцлеРА и возможный новый подходк к сильной двоїственности для задач оптиматььночо управ-. ления с кратными интегралами.

A duality is presented for problems of optimal control with multiple integrals. In the centre of interest the question on strong duality stands. The mean idea is to study the bi-dual problem to the given optimal control problem. It succeeds to get relations between flow problems in, the sense of YouNG and KLötzLER and to find a new possible approach to assertions on strong duality for control problems with multiple integrals.

\section{Einleitung}

Für die theoretische und numérische Behandlung von Optimierungsproblemen spielt die Dualität eine bedeutende Rolle. Ist ein reelles Funktional $f$ über einer Menge $X$ und das primale Problem

$$
f(x) \rightarrow \inf , \quad x \in X,
$$

gegeben, dann ist

$$
g(y) \rightarrow \sup , \quad y \in Y,
$$

für ein reelles Funktional $g$ über einer Menge $Y$ eine zu ihm duale Aufgabe, wenn

$$
\sup \{g(y) \mid y \in Y\} \leqq \inf \{f(x) \mid x \in X\}
$$

gilt. Insbesondere sprechen wir von starker Dualität zwischen (1) und (2), wenn in (3) die Gleichheit gilt. Bekanntlich kann man mittels der Dualität einerseits Fehlerabschätzungen, andererseits hinreichende Optimalitätsbedingungen für die Aufgabe (1) gewinnen. Dazu ist von besonderem Interesse zu klären, wann zwischen (1) und (2) starke Dualität vorliegt.

Wir untersuchen Steuerungsprobleme und deren entsprechende Dualprobleme im Sinne von R. KLötzler [7] auf starke Dualität. Für Steuerungsprobleme einfacher Integrale und deren zugeordnete Dualprobleme ist diese Frage weitgehend geklärt 
$[8,11,13]$. Dabei spielt im eindimensionalen Falle das Studium der Bellmanschen Funktion eine entscheidende Rolle. Eine solche Funktion steht aber bei mehrdimensionalen Problemen nicht mehr zur Verfügung. In dieser Arbeit wird ein möglicher neuer Zugang zu starken Dualitätsaussagen angegeben. Die grundlegende Idee besteht darin, die duale Aufgabe erneut zu dualisieren. Die gewonnene biduale Aufgabe ist ein Flußproblem im Sinne von L. C. Young [14]. Es gelingt nun, einerseits Beziehungen zwischen diesem sehr abstrakten FluBproblem und einem anschàulich interpretierbaren und relevanten Flußproblem im Sinne von R. KLÖTzLER $[9,10]$ herzustellen und andererseits im eindimensionalen Falle auch den Zusammenhang von Flußproblemen und entsprechenden Steuerproblemen herzustellen. Es ist zu vermuten, da $B$ die hier verwendeten Methoden im wesentlichen auf mehrdimensionale Probleme übertragbar sind. Gelingt dies, so kann die Frage nach starker Dualität im mehrdimensionalen' Fall ohne die bisher allzu einschränkenden Konvexitätsforderungen [3] positiv beantwortet werden.

\section{Problemstellung}

Es sei das folgende Steuerproblem $\left(\mathbf{P}_{0}\right)$ in Dieudonné-Rasherskyscher Form gegeben:

$$
J(x, u)=\int_{0} r(t, x(t), u(t)) d t \rightarrow \operatorname{Min} !
$$

bez. aller $x \in W_{\infty}^{1, n}(\Omega)$ und $u \in L_{\infty}^{r}(\Omega)$, die den

Zustandsgleichungen $x_{t_{a}}(t)=g_{\alpha}(t, x(t), u(t))$ f. ü. auf $\Omega$,

Phasenbeschränkungen $x(t) \in \bar{G} \subset \mathbf{R}^{n}$ auf $\Omega$,

Steuerbeschränkungen $u(t) \in U \subseteq \mathbf{R}^{r}$ f. ü. auf $\Omega$

Randbedingungen $x(s) \in M_{s} \subset \bar{G}$ auf $\partial \Omega$

$(\partial \Omega$ sei der Rand von $\Omega$ ) genügen. Wir treffen folgende Grundvoraussetzungen:

1. $r$ sei stetig und größer Null auf $\bar{\Omega} \times \bar{G} \times U$.

2. $g_{\text {ia }}$ sei stetig auf $\bar{\Omega} \times \vec{Q} \times U$ und $g_{\mathrm{a}}=\left(g_{1 \alpha}, \ldots, g_{n a}\right)^{\mathrm{T}}(i=1, \ldots, n ; \alpha=1, \ldots, m)$.

3. $U \subseteq \mathbf{R}^{r}$ sei kompakt und nicht leer.

4. $\mathfrak{M}_{s}$ sei abgeschlossen und nicht leer für alle $s \in \partial \Omega$.

5. $\Omega \subset \mathbf{R}^{m}$ und $G \subset \mathbf{R}^{n}$ seien Lipschitzgebiete im Sinne von C. B. MorRey und S. HrLdebRandt [5].

6. Die Menge $X_{0}$ aller zulässigen Prozesse $(x, u)$ zu $\left(\mathbf{P}_{0}\right)$ sei nicht leer.

Bezeichnet $\mathfrak{n}(s)$ den äußeren Normalen-Einheitsvektor zu $s \in \partial \Omega$, so erhalten wir als duale Aufgabe zu $\left(\mathbf{P}_{0}\right)$ nach R. KLöTzLER [7] die folgende A afgabe $\left(\dot{\mathbf{D}}_{0}\right)$ :

$$
L(S)=\int_{\partial \Omega} \sigma(s) \mathrm{n}(s) d o \rightarrow \operatorname{Max} !
$$

bez. aller $S \in \mathfrak{S}_{0}$

$$
\begin{aligned}
\mathfrak{S}_{0}= & \left\{S \in \mathcal{S} \mid \nabla_{t} \mathrm{~T}^{\mathrm{T}}(t, \xi)+\left[\nabla_{\xi} S^{\alpha}(t, \xi)\right]^{\mathrm{T}} g_{\alpha}(t, \xi, v) \leqq r(t, \xi, v)\right. \text { auf } \\
& \Omega \times G \times U\} \quad \text { (über doppelte Indizes wird summiert) }
\end{aligned}
$$

mit

$$
\circlearrowleft=\left\{S=\left(S^{1}, \ldots, S^{m}\right) \in C^{1, m}(\bar{\Omega} \times \bar{G}) \mid S(s, \xi)_{\xi \in \mathfrak{M},}=\sigma(s) \text { auf } \partial \Omega\right\} .
$$

Die zwischen $\left(\mathbf{D}_{0}\right)$ und $\left(\mathbf{P}_{\mathbf{0}}\right)$ ausgedrückte Dualität ist nicht involutorisch, wie man schon an der unterschiedlichen Struktur der beiden Probleme erkennen kann. 
Wir können $\left(\mathbf{D}_{0}\right)$ als Aufgabe der linearen Optimierung in Banachräumen $X, Y$ auffassen, die gemäß [4:S. 123] für ein lineares Zielfunktional $\left\langle c^{*}, \cdot\right\rangle_{X}$ auf $X$ lautet:

$$
\left\langle c^{*}, x\right\rangle_{X} \rightarrow \operatorname{Max} !
$$

bez. aller $x \in X$; die den Nebenbedingungen

$$
A x \underset{K(Y)}{\leqq} \text {, und } x \underset{K(X)^{\prime}}{\geqq} 0
$$

genügen. Dabei ist $A: X \rightarrow Y$ ein linearer stetiger Operator, $K(X) \subset X$ und $K(Y)$ $\subset Y$ sind Ordnungskegel. In unserer Aufgabe ist $X=€$ und als Teilraum von $C^{1, m}(\bar{\Omega} \times \bar{G})$ mit der Norm $\|S\|=\sum_{\alpha=1}^{m}\left\|S^{\alpha}\right\|$,

$$
\left\|S^{\alpha}\right\|=\operatorname{Max}_{\bar{\Omega} \times \bar{G}}\left|S^{\alpha}(t, \xi)\right|+\sum_{j=1}^{m} \operatorname{Max}_{\bar{\Omega} \times \bar{G}}\left|S_{t,}^{\alpha}(t, \xi)\right|+\sum_{i=1}^{\operatorname{na}} \operatorname{Max}_{\bar{Q} \times \bar{G}}\left|S_{\xi^{\prime}}^{\alpha}(t, \xi)\right|,
$$

wieder cin Bariachraum; $Y=C(Q)$ in der Maximumnorm mit $Q=\bar{\Omega} \times \bar{G} \times U$, $K(C(Q))=\{f \in C(Q) \mid f(x) \geqq 0$ für alle $x \in Q\}$ und $K(\Theta)=\Subset$. Für $f_{1}, f_{2} \in C(Q)$ soll. $f_{1} \leqq f_{2}$ bez. $K(C(Q))$ bedeuten $f_{2}-f_{1} \in K(C(Q))$ und analog für $S^{\prime} ; S^{\prime \prime} \in \subseteq$ (also ist $S \geqq 0$ bez. $K(\circlearrowleft)$ für alle $S \in \cong$ ). Der Operator $A$ ist folgendermaßen definiert:

$$
A S^{i}=\nabla_{t}{ }^{\mathrm{T}} S^{\mathrm{T}}+\left[\nabla_{\xi} S^{2}\right]^{\mathrm{T}} g_{a} .
$$

Offensichtlich ist er linear, und da $Q$ kompakt ist, folgt

$$
\|A S\|_{Y} \leqq \max \{1, C\}\|S\|_{X}, \quad \operatorname{mit}-C=\max \left\{\left|g_{i \alpha}(t, \xi, v)\right| \mid \text { alle }(t, \xi, v), i, \alpha\right\} .
$$

Damit ist $A$ stetig. Das Zielfunktional $c^{*}$ auf $\subseteq$ läßt sich durch

$$
\left\langle c^{*}, S\right\rangle=\int_{\partial \Omega} \sigma(s) \mathfrak{n}(s) d o .
$$

definieren. Weiter ist $b=r$.

\section{Eíne zu $\left(D_{0}\right)$ duale Aufgabe}

Dem Problem $\left(\mathbf{D}_{0}\right)$ ordnen wir nach Fenchel-Rockafellarscher Dualitätstheorie, angewandt auf lineare Programme [4: S. 108], das duale Programm

$$
\left\langle y^{*}, b\right\rangle_{\dot{Y}} \rightarrow \text { Min! }
$$

bez. aller $y^{*} \in Y^{*}$ zu, die den Nebenbedingungen

$$
A^{*} y^{*} \underset{K^{*}(X)^{*}}{\geqq} c \text { und } y^{*} \underset{K^{*}\left(Y^{*}\right)}{\geqq} 0
$$

genügen. Hier bezeichnen $X^{*}$ und $Y^{*}$ die zu $X$ bzw. $Y$ topologisch dualen Räume, $K^{*}\left(X^{*}\right)$ und $K^{*}\left(Y^{*}\right)$ die zu $K(X)$ und $K(Y)$ dualen Kegel und $A^{*}: Y \rightarrow X$ den zu $A$ adjungierten Operator. Fiir unser Problem ergibt sich speziell:

$Y^{*}=C^{*}(Q)$, und $y^{*} \geqq 0$ bedeutet $\left.\int f(x) d \mu(x) \geqq 0(f \in K(C(Q)))^{1}\right)^{1}$. Ferner ist, da mit $\Phi$ auch $-\Phi$ in $\Subset$ liegt,

$$
K^{*}\left(\bigodot^{*}\right)=\left\{\Phi^{*} \in \Im^{*} \mid\left\langle\Phi^{*}, \Phi\right\rangle=0 \text { für alle } \Phi \in \subseteq\right\}
$$

1) $\mu$ bezeichnet dis durch $y^{*}$ eindehtig bestimmte nichtnegative reguläre Borelsche $\mathrm{MaB}$. 
Folglich heißt $A^{*} y^{*} \geqq c^{*}, \operatorname{da} \beta\left\langle A^{*} y^{*}, \Phi\right\rangle=\left\langle c^{*}, \Phi\right\rangle$ für alle $\Phi \in €$ ist, hzw. nach Definition von $A^{*}$, da $\left\langle c^{*}, \Phi\right\rangle=\left\langle y^{*}, A \Phi\right\rangle$, d. h. ।

$$
\int_{Q}\left\{\nabla_{\imath} \mathbf{T} \Phi \mathbf{T}(t, \xi)+\left[\nabla_{\xi} \dot{\Phi}^{\alpha}(t, \xi)\right]^{\mathrm{T}} g_{a}(t, \xi, v)\right\} d \mu(t, \xi, v)=\int_{\partial \Omega} \varphi(s) \mathfrak{n}(s) d o
$$

für alle $\Phi=\left(\Phi^{1}, \ldots, \Phi^{m}\right) \in \Theta, \varphi(s)=\left.\Phi(s, \xi)\right|_{\xi \in \mathbb{R} \text {, }}$ ist. Schließlich gilt die Darstellung

$$
\left\langle y^{*}, b\right\rangle=\int_{0} r(t, \xi, v) d \mu(t, \xi, v) \text {. }
$$

Damit lautet die zu $\left(\mathbf{D}_{0}\right)$ gemäß Fenchel-Rockafellarscher Theorie dualisierte Aufgabe $\left(\mathbf{D}_{0}\right)^{\mathbf{D}}$ :

$$
\dot{y}^{*}(r)=\int_{0} r(t, \xi, v) d \mu(t, \xi, v) \rightarrow \operatorname{Min} !
$$

bez. aller $y^{*} \in K^{*}\left(C^{*}(Q)\right)$, die für alle $\Phi \in \mathbb{S}$ der Bedingung

$$
\int_{0}\left\{\nabla_{t}{ }^{\mathrm{T}} \Phi^{\mathrm{T}}(t, \xi)+\left[\nabla_{\xi} \Phi^{\alpha}(t, \xi)\right]^{\mathrm{T}} g_{a}(t, \xi, v) d \mu(t, \xi, v)=\int_{\partial \Omega} \varphi(s) \mathfrak{n}(s) d o\right.
$$

genügen.

Ausgehend von der Aufgabe $\left(\mathbf{P}_{0}\right)$ für den Fall $m=1$, also für eindimensionale Steuerungsprobleme, wird bei L. C. Young [14: Kap. 6] und bei R. B. VinTer und R. M. Lewis [13] durch Einbettung der zu $\left(\mathbf{P}_{0}\right)$ zulässigen Prozesse in eine Menge linearer stetiger Funktionale über $C(Q)$ ein ähnliches Problem gewonnen. Wir könnten deshalb in Anlehnung an die zitierten Arbeiten $\left(\mathbf{D}_{0}\right)^{\mathbf{D}}$ als Flußproblem im Sinne von L. C. Young bezeichnen.

Im Sinne der Rockafellarschen Dualitätstheorie zeigen wir nun die Stabilität von $\left(\mathbf{U}_{0}\right)$.

Satz 1: Es existiere ein $\bar{S} \in \bigodot_{0}$ mit $A \bar{S}<r, d$. h. mit

$$
\nabla_{t}^{\mathrm{T}} \overline{\mathcal{S}}^{\mathrm{T}}(t, \xi)+\left[\nabla_{\xi} \bar{S}^{\alpha}(t, \xi)\right]^{\mathrm{T}} g_{a}(t, \bar{\xi}, v)<r(t, \xi, \dot{v}) \quad((t, \xi, v) \in Q)
$$

d. h. $\Im_{0}$ besitze einen inneren Punkt). Dann ist $\left(\mathbf{D}_{0}\right)$ stabil.

Beweis: Nach [4: Satz 6] ist $\left(\mathbf{D}_{0}\right)$ stabil, wenn $f: C(Q) \rightarrow \mathbf{R}$ mit

$$
f(y)=\left\{\begin{array}{cl}
0 & \text { für } y \leqq r \\
-\infty & \text { sonst }
\end{array}\right.
$$

an der Stelle $A \bar{S}$ stetig ist. Diese Forderung ist erfüllt, wen'́n $A \bar{S}$ ein innerer Punkt von $\Im_{0}$ ist und die Stetigkeit von $f$ aus der Konvexität von $-f$ folgt. Das wiederum. ist erfüllt nach [4: Satz 24], da $-f$ ein konvexes unterhalbstetiges Funktional auf einem Banachraume ist

Für den weiteren Verlauf der Arbeit werden wir $\left(\mathbf{D}_{0}\right)$ stets als stabil voraussetzen. Die damit gewonnene Aussage über starke Dualität zwíischen $\left(\mathbf{D}_{0}\right)$ und $\left(\mathbf{D}_{0}\right)^{\mathrm{D}}$ erlaubt_uns, die Aufgabe $\left(\mathbf{P}_{0}\right)^{\prime}$ in die sehr abstrakte Aufgabenstellung $\left(\mathbf{D}_{0}\right)^{10}$ einzubetten:

$$
\sup \left(\mathbf{D}_{0}\right)=\min \left(\mathbf{D}_{0}\right)^{\mathbf{D}} \leqq \inf \left(\mathbf{P}_{\mathbf{0}}\right)
$$

und wie man leicht zeigen kann, wird durch jedes $(\hat{x}, \hat{u}) \in X_{0}$ ein zulässiges Element $g^{*}$ zu $\left(\mathbf{D}_{\mathbf{0}}\right)^{\mathrm{D}}$ definiert :

$$
\hat{y}^{*}(f)=\int_{0} f(t, \xi, v) d \mu(t, \xi, v)=\int_{0} f(t, \hat{x}(t), \hat{u}(t)) d t \quad(f \in Q)
$$


Im eindimensionalen Falle gilt bei Konvexität von $r(t, \xi, \cdot), g(t, \xi, \cdot)$ und $U$ zwischen $\left(\mathbf{P}_{0}\right)$ und $\left(\mathbf{I}_{0}\right)$ starke Dualität, weshalb dann $\min \left(\mathbf{D}_{0}\right)^{\mathrm{D}}=\inf \left(\mathrm{P}_{0}\right)$ ist. Unser Ziel ist es, unter ähnlichen Voraussetzungen solch ein Resultat auch im mehrdimensionalen Falle nach. zuweiseǹ.

\section{Flüsse}

Um eine anschauliche Vorstellung von „Flüssen“ im Sinne von L. C. Youvg zu gewinnen, führen wir nun in Anlehnung an [9, 10] Flußprobleme im Sinne von R. KLötzLER ein. Dabei sei zunächst folgende Begriffsbildung vorgenommen.

Definition 1 : Es heißt $\mathfrak{w}=v_{0}\left(\begin{array}{c}E_{m} \\ w\end{array}\right)=\left(\mathfrak{w}_{1}, \ldots, \mathfrak{w}_{m}\right)$, mit

$$
\text { - } \mathfrak{w}_{\alpha}=\left(0, \ldots, \underset{\hat{a}}{1}, \ldots, 0, w_{1 \alpha}, \ldots, w_{n a}\right)^{\mathrm{T}} \in C^{1, m+n}(\bar{\Omega} \times \bar{G})
$$

für $\alpha=1, \ldots, m$, Flu $\beta$ über $U$, wenn $v_{0} \geqq 0$ auf $\bar{\Omega} \times \bar{G}$ und für $j=1, \ldots, n$

$$
w_{j \alpha}(t, \xi)=\int_{y} g_{j \alpha}(t, \xi, v) d \mu_{(\ell, \xi)}(v)^{t} \text { f. } \ddot{\mathrm{u}} \text {. auf } \bar{\Omega} \times \bar{G}
$$

gilt. Dabei ist $\left\{\mu_{(t, \xi)} \mid(t, \xi) \in \widehat{\Omega} \times \bar{G}\right\}$ eine Familic von Wahrscheinlichkeitsmaßen mit den folgenden Eigenschaften:

(A) Für jedes $f \in C(\bar{\Omega} \times \dot{\bar{G}} \times U)$ ist nachfolgende Funktion L-meßbar :

$$
h_{f}: \bar{\Omega} \times \dot{\bar{G}} \rightarrow \mathbf{R} \text { mit } h_{f}(t, \xi)=\int_{U} f(t, \xi, v) d \mu_{(t, \xi)}(v) .
$$

(B) Auf $\left\{\xi \in G \mid v_{0}(t, \xi)>0\right\}$ ist nachfogende Funktion stetig :

$$
r_{\mathfrak{p}}(t, \cdot) \text { mit } r_{\mathfrak{w}}(t ; \xi)=\int_{U} r(t, \dot{\xi}, v) d \mu_{(t, \xi)}(v)(t \cdot \epsilon \Omega) .
$$

Die Menge aller Flüsse über $U$ bezeichnen wir mit $\mathfrak{R}_{U}$ und formulieren folgendes Flußproblem (F) (in [9] als Flußproblem 1. Art bezeichnet):

$$
F(\mathfrak{w})=\int_{0} \int_{G} \int_{U} r(t, \xi, v) d \mu_{(t, \xi)}(v) v_{0}(t, \xi) d \xi d t \rightarrow \text { Min! }
$$

bez. aller $\mathfrak{w} \in \mathfrak{A}_{U}$, die den Nebenbedingungen

$$
\begin{array}{ll}
\left.\bar{\nabla}_{(t, \xi)}^{\mathrm{r}} \mathfrak{w}_{a}=0^{2}\right) & \text { auf } \Omega \times G^{\prime}(\alpha=1, \ldots, m), \\
\int_{\mathfrak{Q} l_{a}} v_{0}(s, \xi) d \xi \doteq 1 & \text { auf } \partial \Omega
\end{array}
$$

und

mit

$$
\left.\mathfrak{w}_{\mathfrak{a}}^{\mathbf{T}} d \mathfrak{v}_{\bar{\Omega} \times \bar{G}}=0^{3}\right) \quad \text { auf } \partial(\bar{\Omega} \times \bar{G}) \backslash \dot{\mathfrak{U}}
$$

$$
\mathfrak{H}=\left\{(s, \xi) \in \mathbf{R}^{m} \times \dot{R}^{n} \mid s \in \partial \Omega \text { und } \xi \in \mathfrak{M} \mathbb{R}_{s}\right\} .
$$

genügen. Die Menge der zulässigen Flüsse zu (F) bezeichnen wir mit $Y_{0}$.

2) $\nabla_{t, \xi}^{\mathrm{T}}=\left(\frac{\partial}{\partial t_{1}}, \ldots, \frac{\partial}{\partial t_{m}}, \frac{\partial}{\partial \xi_{1}}, \ldots, \frac{\partial}{\partial \xi_{n}}\right)$.

$\left.{ }^{3}\right) \mathrm{do} \bar{Q} \times \bar{G}$ bezeichnet das im $\mathbf{E}^{m+n}$ nach außen orientierte vektorielle Oberflächenelement $z, u$ $\partial(\bar{\Omega} \times \bar{G})$. 
Die durch Flüsse iv $\in Y_{0}$ erzeugten linearen stetigen Funktionale $y^{*}$ auf $C(Q)$ mit

$$
y^{*}(f)=\int_{Q} f(t, \xi, v) d \nu(t, \xi, v):=\iint_{\Omega \cdot G} \int_{U} f(t, \xi, v) d \mu_{(t, s)}(v) v_{0}(t, \xi) d \xi d t
$$

erfüllen die Bedingung (4), denn nach Gaußschem Integralsatz folgt wegen (5), (7) und (8)

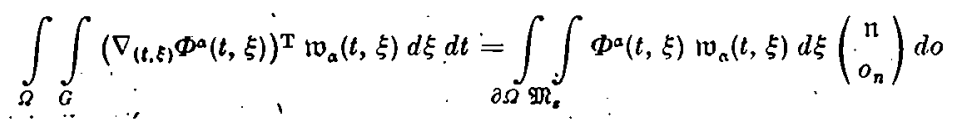

mit $\mathfrak{n}$ als äußeren Normalen-Einheitsvektor zu $\partial \Omega$ und $o_{n}$ als $n$-dimensionalen Nullvektor. Schließlich gilt nach (6) und Definition von $\Phi$

$$
\int_{\Omega} \int_{G}\left(\nabla_{(t, \xi)} \Phi^{\alpha}(t, \xi)\right)^{\mathbf{T}} \mathfrak{w}_{\alpha}^{\prime}(t, \xi) d \xi d t=\int_{\partial \Omega} \varphi(s) \mathfrak{n}(s) d o .
$$

Durch Flüsse $\mathfrak{w} \in Y_{0}$ werden also zulässige Elomente $z u\left(\mathrm{I}_{0}\right)^{\mathrm{D}}$ erzeugt, deshalb gilt dic Relation $\min \left(\mathbf{D}_{0}\right)^{\mathrm{D}} \leqq \inf (\mathbf{F})$. Ist umgekehrt $\mathfrak{w} \in \mathfrak{\Re}_{U}$ und gilt (4), so folgt, da $B$ w die Nebenbedingungen (5) $-(8)$ erfüllt [9].

Die Interpretation des Flusses nehmen wir der_Einfachbeit halber für den Fall eindimensionaler Probleme $(m=1)$ vor. Ein Fluß.w $\in . \Re_{U}$ ist zulässig bezüglich (F), wenn er in $\left\{t_{0}\right\} \times \mathfrak{M}_{t_{0}}$, mit einer bestimmten Intensität $\int_{\mathbb{N}_{0}} v_{0}\left(t_{0}, \xi\right) d \xi .(=1)$ startet; in $\left(t_{0}, t_{1}\right) \times G$ quellfrei verläuft und dabei gewisse Richtungsbeschränkungen und Zustandsgleichungen einhält und mit der gleichen Intensität in $\left\{t_{1}\right\} \times \mathcal{M}_{l_{1}}^{\prime}$ ankommt. Ein Flußproblẹm dieser Gestalt entsteht aus einem Steuerproblem $\left(\dot{\boldsymbol{P}}_{0}\right)$ bei konvexem $U$ und für alle $(t, \xi) \in\left[t_{0}, t_{1}\right] \times \bar{G}$ konvexen $g_{i}(t, \xi, \cdot)(i=1, \ldots, n)$ und $r(t, \xi, \cdot)$, wenn anstelle der zulässigen Trajektorien zu $\left(\mathbf{P}_{0}\right)$, die "wir als",fadenförmig dünne" Flüsse auffassen können, ,breitere" Flüsse vo $\in Y_{0}$ zugelassen $\because$ werden.

Wir wollen nun den Zusammenhang źwischen dem Problem $\left(\mathbf{D}_{0}\right)^{\mathrm{D}}$ und dem Flußproblem (F) näher charakterisieren.

Definition 2: Eine Folge von Flïssen $\left\{\mathfrak{w}^{n}\right\} \subset \mathfrak{R}_{U}$ heißt asymptolisch zulässig bez. $Y_{0}$, wenn für alle $\Phi \in \dot{\mathscr{S}}$ gilt

$$
\lim _{n \rightarrow \infty} \int_{\Omega} \int_{G}\left\{\nabla_{t} \mathbf{T} \Phi^{\mathbf{T}}(t, \xi)+\left[\nabla_{\xi} \Phi^{\alpha}(t, \cdot \xi)\right]^{\mathbf{T}} w_{a}^{n}(t, \xi)\right\} v_{0}{ }^{n}(t, \xi) d \xi d t=\int_{Q} \varphi(s) \mathfrak{n}(s) d o .
$$

Satz 2: $Z u$ jedem zulässigen Element $y^{*} \in C^{*}(Q)$ zum Problem $\left(\mathbf{D}_{0}\right)^{\mathrm{D}}$ existiert eine Folge $\left\{\mathfrak{w}^{n}\right\} \subset \Re_{U}$ asymptotisch zulässiger Flüsse bez. $Y_{0}$, so daß gilt

$$
\lim _{n \rightarrow \infty} \int_{Q} \int_{G} \int_{U} r(t, \xi, v) d \mu_{(t, \xi)}^{n}(\dot{v}) v_{0}^{n}(\dot{t}, \xi) d \xi d t=\int_{Q} r(\dot{t}, \dot{\xi}, v) d \nu(t, \xi, v) .
$$

Beweis: Nach [4: S. $66 \mathrm{ff}$.$] ist C^{*}(Q)$ mit der schwachen* Topologie $\sigma^{*}$ ein lokalkonvexer Hausdorffscher Raum. Wir zeigen, daß die Menge $\hat{Y}^{*}$ aller durch Flüsse $\mathfrak{w} \in \mathfrak{R}_{U}$ erzeugten linearen stetigen Funktionale $y^{*}$, mit

$$
y^{*}(f)=\int_{\Omega} \int_{G} \int_{U} f(t, \xi, v) d \mu_{(t, \xi)}(v) v_{0}(t, \xi) d \xi d t \quad(f \in C(Q)),
$$

in $C^{*}(Q)$ bez. dieser Topologie dicht liegt.

1. $\hat{Y}^{*}$ ist konvex: Sind $y_{1}^{*}, y_{2}^{*} \in \hat{Y} \hat{Y}^{*}$, so gilt

$$
y_{l}^{*}(f)=\int_{\Omega} \int_{G} \int_{V} f(t, \xi, v) d \mu_{(t, \xi)}^{(}(v) v_{0}^{l}(\dot{t}, \xi) d \xi d t
$$


nit

$$
\mathfrak{w}^{l} \in \Re_{U}^{\prime}, \quad \mathfrak{w}^{l}=v_{0}{ }^{l}\left(\begin{array}{c}
E_{m} \\
w^{l}
\end{array}\right), \quad w_{j a}^{\ell}(l, \xi)=\int_{U} g_{j a}(l, \xi, v) d \mu_{(\ell, \xi)}^{l}(v)
$$

$(l=1,2 ; j=1, \ldots, n ; \alpha=1, \ldots, m)$. Dann ist für $0<\lambda<1$

$$
\left[\lambda y_{1}^{*}+(1-\lambda) y_{2}^{*}\right](f)=\iint_{\Omega} \int_{U} f(t, \xi, v) d \mu_{(t, \xi)}(v) v_{0}(t, \xi) d \xi d t
$$

wobei $\mu_{(t, 5)}$ für jedes $f \in C(U)$ folgendermaßen erklärt sei :

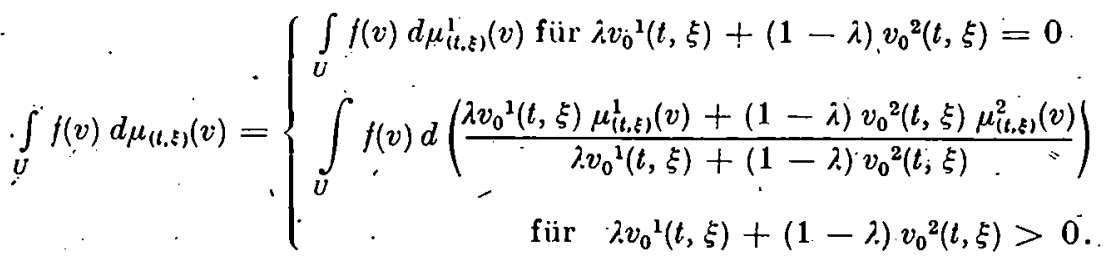

Es gilt:

a) $v_{0} \doteq \lambda v_{0}^{1}+(1-\lambda) v_{0}^{2} \in C^{1}(\bar{\Omega} \times \bar{G})$ und $v_{0}(t, \xi) \geqq 0$ für alle $(t, \xi) \in \bar{\Omega} \times \bar{G}$.

b) $\mu_{(t, \xi)} \geqq 0, \operatorname{supp} \mu_{(t, \xi)} \subseteq U$ und $\int_{U} d \mu_{(t, \xi)}(v)=1$ für f. a. $(t, \xi) \in \Omega \times G$, da $\mu_{(t, \xi)}^{1}$

und $\mu_{(t, \xi)}^{2}$ für f. a. $(t, \xi) \in \Omega \times G$ Wahrscheinlichkeitsmaße sind.

c) Fuir. jede Funktion $f \in C(Q)$ ist

$$
h_{f}: \bar{\Omega} \times \bar{G} \rightarrow \mathbf{R} \text {. mit } h_{f}(t, \xi)=\int_{U} f(t, \xi, v) d \mu_{(t, \xi)}(v)
$$

L-meßbar, da die den Familien $\left\{\mu_{(t, \xi)}^{1}\right\}$ und $\left\{\mu_{(t, \xi)}^{2}\right\}$ entsprechenden Funktionen $h$ diese Eigenschaft (A) haben. Ebenso ist

$$
r_{\mathfrak{r v}}(t, \cdot) \text { mit } \quad r_{\mathfrak{r}}(t, \xi)=\int_{U} r(t, \xi, v) d \mu_{(t, \xi)}(v)
$$

in $\left\{\xi, \epsilon G \mid \lambda v_{0}^{1}(t, \xi)+(1-\lambda) v_{0}^{2}(t, \xi)>0\right\}$ stetig (Eigenschaft (B)).

Also ist $\mathfrak{w}=\left(\mathfrak{w}_{1}, \ldots, \mathfrak{w}_{m}\right)$ ein Flu $\beta$ über $U$, der das Funktional $\lambda y_{1}{ }^{*}+\left(1-\lambda_{1}\right) y_{2}{ }^{*}$ erzeugt. Folglich ist letzteres ein Element von $G^{*}$ und diese Menge konvex.

2. Wir wenden nun folgenden Trennungssat\%. [4: S. 71] an:: $X$ sei ein lokalkonvexer Hausdorffscher Raum und $M \subset X$ eine dessen Nullelement enthaltende konvexe abgeschlossene Teilmerige, $x^{0} \in X \backslash M$. Dann existiert ein $x^{*} \in X^{*}$. mit $\cdot\left\langle x^{*}, x^{0}\right\rangle>1$ und $\left\langle x^{*}, x\right\rangle \leqq 1$ für alle $x \in M$. Wir wenden dies auf $C^{*}(Q)$ mit der schwachen* Topologie $\sigma^{*}$ und $M$ gleich dem Abschluß von $\hat{Y}^{*}$ in $\sigma^{*}$ an. Würde ein $\bar{y}^{*} \in K^{*}\left(C^{*}(Q)\right) \backslash M$ existieren, so gäbe es ein $f \in\left[C^{*}(Q), \sigma^{*}\right]^{*}$ mit

$$
\bar{y}^{*}(\hat{f})=\int_{0} \hat{f}(t, \xi, v) d \hat{\nu}(t, \xi, v)>1
$$

( $\mathcal{D}$ ist das durch $\bar{y}^{*}$, eindeutig bestimmte nichtnegative Borelsche MaßB) und

$$
\int_{\Omega}: \int_{G} f(t, \xi, v) d \mu_{(t, \xi)}(v) v_{0}(t, \xi) d \xi \dot{d} t \leqq 1 \text { für alle } \mu, v_{0}
$$

mit

$$
\mathfrak{w}=v_{0}\left(\begin{array}{c}
E_{m} \\
w
\end{array}\right), \quad w_{j a}(t, \xi)=\int_{U} g_{j a}(t, \xi, v) d \mu_{(t, \xi)}(v), \quad \mathfrak{w} \in \mathfrak{R}_{U}
$$


Wäre $\hat{f} \leqq: 0$, dann ist wegen $\bar{y}^{*} \in K^{*}\left(C^{*}(Q)\right)$-auch $\int_{0} f(t, \xi, v) d \hat{\imath}\left(t, \xi, v^{\prime}\right) \leqq 0$. Also existiert $\operatorname{ein}\left(t^{\prime}, \xi^{\prime}, v^{\prime}\right) \in \operatorname{supp} \nu \operatorname{mit} \hat{f}\left(t^{\prime}, \xi^{\prime}, v^{\prime}\right)>0$. Es sei

$$
\hat{f}\left(t^{0}, \xi^{0}, v^{0}\right)=\max \{\hat{f}(t, \xi, v) \mid(t, \xi, v) \in \operatorname{supp} \text { D }\} \text {. }
$$

Da $f$ stetig und $\Omega \times G$ ein Lipschitzgebiet ist, existiert eine Teilmenge $\dot{\Omega}^{\prime} \times G^{\prime}$ $\leqq \Omega \times G$ mit mes $\left(\Omega^{\prime} \times G^{\prime}\right)>0$, so daß $f\left(t, \xi, v^{0}\right)>0$ für alle $(t, \xi) \in \Omega^{\prime} \times G^{\prime \prime}$ ist. Wählt man nun

$$
\tilde{v}_{0}(t, \xi)= \begin{cases}1 & \text { für }(t, \xi) \in \bar{\Omega}^{\prime} \times \bar{G}^{\prime} \\ 0 & \text { für }(t, \xi) \in \bar{\Omega} \times \bar{G} \backslash\left(\bar{\Omega}^{\prime} \times \bar{G}^{\prime}\right)\end{cases}
$$

und $\mu_{(t, \xi)}=\delta_{v^{\bullet}}$ (dies ist das in $v^{0} \in U$ konzentrierte Diracmaß) für alle $(t, \xi) \in \bar{\Omega} \times \bar{G}$, so erhält man

$$
\int_{\Omega} \int_{G} \int_{U} \hat{f}(t, \xi, v) d \mu_{(t, \xi)}(v)^{\prime} \tilde{v}_{0}(t, \xi) d \xi \dot{d} \iota=\iint_{\Omega^{\prime}} \int_{G^{\prime}} \hat{f}\left(t, \xi, v^{0}\right) d \xi d t=\lambda>0 .
$$

Da $C_{0}{ }^{\infty}(\Omega \times G)$ in $L_{p}(\Omega \times G), p \geqq 1$, dicht liegt, existiert zu $\bar{v}_{0}$ eine Folge $\left\{v_{0}{ }^{k}\right\}$ $\subset C_{0}{ }^{\infty}(\Omega \times G)$, die f. ï. gegen $\bar{v}_{0}$ konvergiert und für die $0 \leqq v_{0}{ }^{k} \leqq \bar{v}_{0}$ für alle $k$ gilt. Für diese Folge ist darin

$$
\lim _{k \rightarrow \infty} \int_{\Omega} \int_{G} \int_{U} f(t, \xi, v) d \delta_{v^{\circ}} v_{0}^{k}(t, \xi) d \xi d t=\lambda
$$

und für beliebiges $\varepsilon \geq 0^{\prime}$ gilt

$$
\lim _{-k \rightarrow \infty} \int_{\Omega} \int_{G} \int_{U} \hat{f}(t, \xi, v) d \delta_{v^{\circ}}(1 / \lambda+\varepsilon) d \xi d t=(1 / \lambda+\varepsilon) \lambda>1 .
$$

Somit existiert ein natürliches $k$ mit

$$
\int_{\Omega} \int_{G} \int_{U}-\hat{f}(t, \xi, v) d \delta_{v^{\circ}}(1 / \lambda+\varepsilon) v_{0}^{k}(t, \xi) d \xi d t>1
$$

und $\mathfrak{w}=v_{0}^{k}(1 / \lambda+\varepsilon)\left(\begin{array}{c}E_{m} \\ w\end{array}\right)$ mit $w_{j a}(t, \xi)=g\left(t, \xi, v^{0}\right)$ ist ein Flu $\stackrel{\leftrightarrow}{\beta}$, der $(9 \mathrm{~b})$ verletzt.

3.' Es' liegt also $\hat{Y}^{*}$ dicht in $K^{*}\left(C^{*}(Q)\right)$. Zu jedem $y^{*} \in K^{*}\left(C^{*}(Q)\right)$ existiert deshalb eine Folge $\left\{\mathfrak{w}^{h}\right\} \subset \Re_{U}$ mit (für alle $f \in C(Q)$ )

$$
\begin{aligned}
& y^{*}(f)=\int_{Q} f(t, \xi, v) d \nu(t, \xi, v)=\lim _{n \rightarrow \infty} \int_{\Omega} \int_{G} \int_{U} f(t, \xi, v) d \mu_{(t, \xi)}^{n}(v) v_{0}^{n}(\dot{t}, \xi) d \xi d t, \\
& \mathfrak{w}^{n}=v_{0}^{n}\left(\begin{array}{c}
E_{m} \\
w
\end{array}\right) \text { und } w_{j a}^{n}(t, \xi)=\int_{U} g_{j a}(t, \xi, v) d \mu_{(t, \xi)}^{n}(v) .
\end{aligned}
$$

Erfüllt $y^{*}$ zusätzlich (4), dann gilt für $f=\nabla_{l} \mathrm{~T} \Phi^{\mathrm{T}}+\left[\nabla_{\xi} \Phi^{\alpha}\right]^{\mathrm{T}} g_{\alpha}$

$$
\begin{aligned}
& \int_{\Omega}\left\{\nabla_{t} \mathbf{T} \Phi \mathbf{T}(t, \xi)+\left[\nabla_{\xi} \Phi^{\alpha}(t, \xi)\right]^{\mathbf{T}} \dot{g}_{a}(t, \xi, v)\right\} d \nu(t, \xi, v)=\int_{\partial \Omega} \varphi(s) \cdot \mathfrak{n}(s) d o \\
& =\lim _{n \rightarrow \infty} \int_{\Omega} \int_{G}\left\{\nabla_{t} \mathbf{T} \Phi^{\mathbf{T}}(t, \xi)+\left[\nabla_{\xi} \dot{\Phi}^{\alpha}(t, \xi)\right]^{\mathbf{T}} w_{\alpha}^{n}(t, \xi) v_{0}^{n}(t, \xi) d \xi d t,\right.
\end{aligned}
$$

d. h. $\left\{\mathfrak{r}^{n}\right\}$ ist asymptotisch zuläșsig. Speziell für $\hat{f}=r$ ist die Behauptung des Satzes erfüllt 
Die Aussage von Satz 2 kann auch als Dualitätsaussage aufgefaBt werden:

$$
\sup \left(\mathbf{D}_{0}\right)=\underline{\lim }\left\{\mathbf{F}\left(\mathfrak{r}^{n}\right) /\left\{\mathfrak{r}^{n}\right\} \text { asymptotisch zulässig zu } f\right\} \text {. }
$$

Es gibt Beispiele, in denen sup $\left(\mathbf{D}_{0}\right)<\inf (\mathbf{F})$ ist (vgl. [10]).

\section{Zusammenhang zwischen zulässigen Flüssen zum FluBproblem}

und zulässigen Prozessen zum Steuerungsproblem im eindimensionalen Fall

- Die Grundgedanken des folgenden Satzes stützen sich wesentlich auf die anschauliche Interpretation eines zulässigen Flusses: Aus jedem Fluß w. $\in Y_{0}$, der mit vorgegeberier Intensität in $\left\{t_{0}\right\} \times 9 \mathcal{J}_{t_{0}}$ startet, in $\left(t_{0}, t_{1}\right) \times G$ quellfrei verläuft und in. $\left\{t_{1}\right\} \times \mathfrak{M}_{i_{1}}$ mit der gleichen Intensität ankommt, kann man einen ,;Stromfaden“ auswählen, der, wenn man die Flüssigkeit mit der gleichen Gesamtintensität durch diesen einen ,Stromfaden“ fließen läßt, dem entsprechenden Zielfunktional einen Wert erteilt, der kleiner oder gleich dem Wert des Zielfunktionals des FluBproblems für den vorgegebenen Fluß ist. Vorbereitend formulieren wir

Hilfssatz 1 : Es sei $\mathfrak{w}=\dot{v}_{0}\left(\begin{array}{c}1 \\ w\end{array}\right) \in \Re_{U}$ ein zulässiger Flu $z u(\dot{\mathbf{F}})$. Dann hat das nachfolgend definierte $\tilde{\mathfrak{w}}=\tilde{v}_{0}\left(\begin{array}{c}1 \\ w\end{array}\right)$ die Eigenschafl

Dabei ist

$$
\left.\int_{\tilde{\mathfrak{M}} t_{t}} \tilde{v}_{0}(t, \xi) d \xi=\int_{\tilde{\mathfrak{M}}_{t_{0}}} v_{0}\left(t_{0}, \xi\right) d \xi \quad\left(t \in t_{0}, t_{1}\right)\right)
$$

$$
\begin{aligned}
& \tilde{v}_{0}(t, \xi)= \begin{cases}v_{0}(t, \xi) / \bar{I} & \text { für }(t, \xi) \in \tilde{G} \\
0 & \text { für }(t, \xi) \in\left\{\left[t_{0}, \dot{t}_{1}\right] \times \bar{G}\right\} \backslash \tilde{G},\end{cases}
\end{aligned}
$$

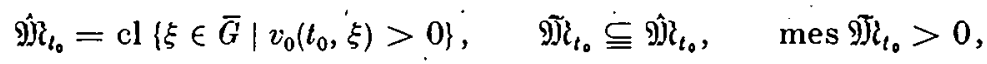

$$
\begin{aligned}
& \tilde{I}=\int_{\tilde{N} l_{0}} v_{0}\left(t_{0}, \xi\right) d \xi \\
& \tilde{G}=\left\{(t, \xi), \in\left[t_{0}, t_{1}\right] \times G \mid \text { es existiert ein } x \text { mit } \dot{x}(\tau)=v(\tau, x(\tau))\right. \\
& \text { f. ü. auf } \left.\left(\iota_{0}, t_{1}\right), x(t)=\xi, x\left(t_{0}\right) \in \mathfrak{\mathfrak { J }} \mathfrak{t}_{t_{0}}\right\}, \\
& \tilde{\mathfrak{J}} \boldsymbol{Z}_{t}=\{\xi \in G \mid(t, \xi) \in \tilde{G}\} .
\end{aligned}
$$

Beweis: Da ro zulässig ist, so gilt, $\nabla_{(t, \xi)}$ wo $=0$ und $v_{0}$ ist folglich bei festem $w$ Lösung der Differentialgleichung

$$
\left(v_{0}\right)_{t}+w^{\mathbf{T}} \nabla_{\xi} v_{0}=-v_{0}\left[\nabla_{\xi}^{\mathbf{T}} w\right]
$$

deren Charakteristiken dem folgenden System genügen :

$$
\left.\begin{array}{l}
\dot{x}_{0}(t)=1 \\
\dot{x}(t)=w\left(x_{0}(t), x(t)\right) \\
\dot{z}(t)=-z(t)\left[\nabla_{\xi}^{\mathrm{T}} w\left(x_{0}(t), x(t)\right)\right] \\
\text { auf }\left(t_{0}, t_{1}\right)
\end{array}\right\} .
$$

bzw. mit $x_{0}(t)=t$

$$
\left.\begin{array}{l}
\dot{x}(t)=w(t, x(t)) \\
\dot{z}(t)=-z(t)\left[\dot{\nabla}_{\xi}^{\mathrm{T}} w(t, x(t))\right] \text { auf }\left(t_{0}, t_{1}\right)
\end{array}\right\} .
$$


Zu $\bar{v}_{0}$ konstruieren wir nun eine Folge $\left\{\tilde{v}_{0}{ }^{n}\right\}$, so dảß bei festem $t \in\left[t_{0}, t_{1}\right]$

$$
\begin{aligned}
& \lim _{n \rightarrow \infty} \tilde{v}_{0}^{n}(t, \xi)=\bar{v}_{0}(t, \xi) \text { für f. a: } \xi_{0} G^{t} \\
& \tilde{\mathfrak{w}}^{n}=\tilde{v}_{0}{ }^{n}\left(\begin{array}{c}
1 \\
w
\end{array}\right) \in C^{1}\left(\left[t_{0}, t_{1}\right] \times \bar{G}\right) \text { und } \nabla_{(t, \xi)}, \tilde{\mathfrak{w}}^{n}=0^{\prime} \text { auf }\left(t_{0}, t_{1}\right) \times G \text {. }
\end{aligned}
$$

gilt. Wir definieren zunächst $\alpha$ nach

$$
\alpha(\eta)=\left\{\begin{array}{ll}
1 & \text { für } \eta \in \widetilde{\mathfrak{N}} t_{0} \\
0 & \text { fïr } \eta \in \bar{G} \backslash \widehat{\mathfrak{J}} t_{t_{0}}
\end{array} .\right.
$$

Dazu existiert eine Folge $\left\{\alpha^{n}\right\} \subset C^{1, n+1}(G)$ mit $\alpha^{n} \leqq \alpha$ und $\alpha^{n}(\eta)=0$ für alle $n$ und $\eta \in \bar{G} \backslash \mathfrak{M T}_{l_{0}}$, die f. ü. (im Sinne des L-Maßes auf $G$ ) gegen $\alpha$ konvergiert. Die Iösung $\psi=\left(\psi_{1}, \ldots, \psi_{n}\right)$ von (11) hängt ḅei stetig differenzierbarer rechter. Seite stetig von den Anfangswerten $\eta=\left(\eta_{1}, \ldots, \eta_{n}\right) \in \bar{G}$ ab und nach [6: S. 175ff.] exi-. stieren die Ableitungen

$$
\psi_{k_{t}}, \psi_{k \eta_{i}}, \ldots, \psi_{k \eta_{i}} \text { und. } \psi_{k \eta_{i} l}=\psi_{k l \eta_{i}} \quad(i, k=1,2, \ldots, n)
$$

und sind stetig. Für die Funktionaldeterminante gilt dabei

$$
\left|\frac{\partial\left(\psi_{1}, \ldots, \psi_{n}\right)}{\partial\left(\eta_{1}, \ldots, \eta_{n}\right)}\right| \neq 0 \text { in }\left(t_{0}, t_{1}\right) \times G
$$

Infolgedessen läßt sich die Lösung von (11) nach den Anfangswerten auflösen: $\eta=\chi(t, \xi)$, und es gilt $\chi \in C^{1, n}\left(\left[t_{0}, t_{1}\right] \times \bar{G}\right)$. Die Funktion $\tilde{v}_{0}^{n}$ mit $\dot{v}_{0}^{n}(t, \xi)=\alpha^{\prime \prime}(\chi(t, \xi))$ $\times \tilde{v}_{0}(t, \xi)$ ist dann Element von $C^{1}\left(\left[t_{0}, t_{1}\right] \times G^{t}\right)$ und in $\left(t_{0}, t_{1}\right) \times G$ genau dann 1 ösung von (10), wenn durch jeden Punkt $(t, \xi) \epsilon \cdot\left(t_{0}, t_{1}\right) \times G$ genau eine Charakteristik geht, die ganz der Fläche $\tilde{v}_{0}{ }^{n}$ angehört, d. h. längs jeder Charakteristik $z-\tilde{v}_{0}{ }^{n}$ konstant ist (vgl. [6: Saţz 1]). Da $v_{0}$ Lösung von (10) ist, gilt $d\left(z(t)-v_{0}(t, x(t))\right) / d t=0$ entlang jeder Charakteristik $(x, z)$. Mit $(x, z)$ ist fiir belicbiges $\lambda>0$ auch $(x, \lambda z)$ Charakteristik, folglich gilt

$$
\frac{d}{d t}\left(\alpha^{n}\left(x\left(l_{0}\right) z(t)-\tilde{v}_{0}^{n}(t, x(t))\right)\right)=\alpha^{n}\left(x\left(t_{0}\right)\right) \frac{d}{d t}\left(z(t)-v_{0}(t, x(t))\right)=0
$$

Damit ist $\tilde{v}_{0}^{n}$ tatsächlich Lösung von (10) und $\tilde{\mathfrak{w}}^{n}=\tilde{v}_{0}^{n}\left(\begin{array}{c}1 \\ w\end{array}\right)$ erfïllt die Gleichung $\nabla_{(t, \xi)} \tilde{\mathfrak{w}}^{n}=0$ in $\left(t_{0}, t_{1}\right) \times G(\dot{n}=1,2, \ldots)$. Auf die Funktionen $\tilde{\mathfrak{w}}^{n}$ wenden wir den Gaußșchen Integralsat $\%$ an : für alle $t \in\left(t_{0}, t_{1}\right)$ ist

$$
\begin{aligned}
& \int_{t_{0}}^{t} \int_{G}\left[\nabla_{(t, \xi)} \Phi(t, \xi)\right]^{\mathrm{T}} \cdot \tilde{\mathbf{w}}^{n}(t, \xi) d \xi d t \\
& =\int_{t_{0}}^{t} \int_{G} \Phi(t, \xi) \nabla_{(l, \xi)} \tilde{\mathfrak{w}}^{n}(t, \xi) d \xi d t+\int_{\left.\partial\left(t_{0}, t\right] \times \bar{G}\right)} \Phi(t, \dot{\xi}) \tilde{\mathfrak{w}}^{n}(t, \xi) d o .
\end{aligned}
$$

Längs $\left.\dot{\partial}\left(\left[t_{0}, t_{1}\right] \times \bar{G}\right) \backslash\left[\left\{t_{0}\right\} \times \mathfrak{M}\right\}_{\ell_{0}} \cup\left\{t_{1}\right\} \times M i_{t_{1}}\right]$ ist $\tilde{w}^{n} d o=\tilde{\mathfrak{w}} d o=0$, demzufolge

und

$$
\int_{\partial\left(\left[t_{0}, t\right] \times \bar{G}\right)} \Phi(t, \xi) \tilde{w}^{n}(t, \xi) d \xi d t=\int_{\mathfrak{D} t_{t}} \Phi(t, \xi) \tilde{v}_{0}^{n}(t, \xi) d \xi-\int_{M_{t_{0}}} \Phi\left(t_{0}, \xi\right) \tilde{v}_{0}^{n}(t, \xi) d \xi
$$

$$
\begin{aligned}
& \int_{t_{0}}^{t} \int_{G}\left[\nabla_{(t, \xi)} \Phi(t, \xi)\right]^{\mathrm{T}} \tilde{\mathfrak{v}}^{n}(t, \xi) d \xi d t \\
& =\int_{\mathbb{R}_{t}} \Phi(t, \xi) \tilde{v}_{0}^{n}(t, \xi) d \xi-\int_{\mathbb{M}_{l_{0}}} \Phi\left(t_{0}, \xi\right) \tilde{v}_{0}^{n}(t, \xi) d \xi .
\end{aligned}
$$


Speziell für $\Phi \equiv 1$ érhalten wir

$$
0=\int_{\mathbb{R}_{t}} \bar{v}_{0}^{n}(t, \xi) d \xi-\int_{\mathfrak{Q} \mathbb{R}_{l_{0}}} \tilde{v}_{0}^{n}(t, \xi) d \xi
$$

Die Folge $\left\{\alpha^{n}\right\}$ konvergiert für f. a. $\eta \in \vec{G}$ gegen $\alpha$. Infolgedessen konvergiert auch die , Folge $\left\{\alpha^{n}(\chi(t, \xi))\right\}$ bei festem $t \in\left(t_{0}, t_{1}\right)$ für f. a. $\xi \in G$ gegen $\alpha(\chi(t, \xi))$. In der Tat, sei a die Menge aller $\xi \in \bar{G}$, für die $\left\{\alpha^{n}(\chi(t, \xi))\right\}$ nicht gegen $\alpha(\chi(t, \xi))$ konvèrgiert, mes $\mathfrak{a}>0$ und $\Omega=\chi(t, \mathfrak{a})$. Dann ist

$$
\operatorname{mes} \mathfrak{L}=\int_{\mathfrak{a}}\left|\frac{\partial\left(\chi_{1}, \ldots, \chi_{n}\right)}{\partial\left(\xi_{1}, \ldots, \xi_{n}\right)}\right| d \xi^{\prime}
$$

und nach (12) mes $\mathfrak{Q}>0$ im Widerspruch zur Voraussetzung, da $\mathbb{B} \alpha^{n}$ für f. a. $\eta \in G$ gegen $\propto$ konvergiert. Damit konvergiert $\left\{\bar{v}_{0}^{n}(t, \xi)\right\}$ bei festem $t \in\left(t_{0}, t_{1}\right)$ für f. a. $\xi \in G$ gegen $\alpha(\chi(t, \xi)) v_{0}(t, \xi)=\tilde{v}_{0}(t, \xi)$ und nach dem Lebesgueschen Grenzwertsatz [1: Satz 13.8.4/S. 138] erhält man aus (13) für f. a. $t \in\left[t_{0}, t_{1}\right]$ durch Grenzübergang $n \rightarrow \infty$

$$
0^{\prime}=\int_{\mathrm{M}_{t}} \tilde{v}_{0}(t, \xi) d \xi-\int_{\mathrm{yi}_{l_{0}}} \tilde{v}_{0}\left(t_{0}, \xi\right) d \xi \quad \mathrm{bzw} . \quad \tilde{I}=\int_{\tilde{\mathrm{M}}_{\ell}} \tilde{v}_{0}(t, \xi) d \xi
$$

Formel (14) gilt zunächst nach [12: S. 263ff.] für beliebige Intervallbereiche a und damit auch für jede Borelmenge. Aus der Regularität des Lebesgueschen Maßes kann man nún leicht ¿schlußfolgern, daß sie' dann auch für jede $L$-meßbare Menge gilt.

Satz 3: Sind die Grundvoraussetzungen 1-6 für das Problem $\left(\mathbf{P}_{0}\right)$ erfüllt, $U$ konvex, $r(t, \xi, \cdot)$ und $g(t, \xi, \cdot)$ konvex für alle $(t, \xi) \in\left[t_{0}, t_{1}\right] \times \bar{G}$ und mes $]_{t_{0}}>0, \dot{s} \dot{0}$ existiert zu jedem zulassigen Flu $\beta$ wo $\in Y_{0}$ ein zulässiger Proze $\beta(\hat{x}, \hat{u}) \in X_{0}$, mit $F(\mathfrak{w})$ $\geqq J(\hat{x}, \hat{u})$.

Beweis: Wir wählen $a>0$ so, daß gilt

$$
\hat{\mathfrak{\jmath}}_{\mathrm{l}_{0}} \leqq K:=\left\{\xi \in \mathbf{K}^{n} \mid-a \leqq \xi^{l} \leqq a(l=1, \ldots, \dot{n})\right\} .
$$

Die Zerlegung des Intervalls $[-a, a]$ in $2^{i}(i=1,2, \ldots)$ Teilintervalle $\hat{l}_{j}^{i}(j=1$, $\therefore, 2^{i}$ ) gleicher Länge $a / 2^{i-1}$,

$$
\hat{l}_{j}^{i}=\left\{\vartheta \in \mathbf{R} \mid-a+(j-1) a / 2^{i-1} \leqq \vartheta \leqq-a+j a / 2^{i-1}\right\}
$$

niennen wir Zerlegung $i$-ter Ordnung. Das kartesische Produkt vori Intervallen $\hat{L}_{j}^{i}$, $\ldots, \hat{l}_{j}^{i}$, gleicher Ordnung $i$ bildet einen Würfel $i$-ter Ordnung mit der Kantenlänge $a / 2^{i-1}$. Jeder Würfel $(i-1)$-ter Ordnung $K_{j}^{i-1}$ kann als Vereinigung von Würfeln $i$-ter Ordnung dargestellt werden. Zu einem gegebenen Fluß $\mathfrak{k}=v_{0}\left(\begin{array}{c}1 \\ w\end{array}\right) \in \dot{Y}$ kon-' struieren wir nun induktiv eine Folge $\left\{\tilde{\mathfrak{w}}^{i}\right\}_{i=0}^{\infty} \subset L_{n+1}^{\infty}\left(\left(t_{0}, t_{1}\right) \times G\right)$,flußähnlicher" Elemente.

1. $\left.\tilde{\mathfrak{w}}^{0}=v_{0}\left(\begin{array}{c}1 \\ w\end{array}\right)=v_{0}^{0}\left(\begin{array}{l}1 \\ w\end{array}\right), 9\right) l_{t_{0}}^{0}=\operatorname{cl}\left\{\xi \in \bar{G} \mid v_{0}\left(t_{0}, \xi\right)>0\right\}, G^{0}=\left[t_{0}, t_{1}\right] \times \bar{G}$.

2. Sei eine Zerlegung $i$-ter. Ordnung gegeben, $K^{0}=K_{1}^{i} \cup \cdots \cup K_{2^{n_{4}}}^{i}$ und

$$
J^{i}=\left\{j \in\left\{1, \ldots, 2^{n_{1}} \mid \text { 'mes }\left(9 M_{i_{0}}^{i-1} \cap K_{j}^{i}\right) \neq 0\right\} .\right.
$$


Dann definieren wir

wobei

$$
v_{0 . j}^{i}(t, \xi)=\left\{\begin{array}{lll}
v_{0}^{i-1}(t, \xi) / I_{j}^{i} & \text { für }(t, \xi) \in G_{j}^{i}, & j \in J^{i} \\
0 & \text { für }(t, \xi) \in G^{0} \backslash G_{j}^{i}, & j \in J^{i} \\
0 & \text { für }(t, \xi) \in G^{0}, & j \notin J^{i}
\end{array}\right.
$$

$$
I_{j}^{i}=\int_{\mathfrak{N}_{\ell_{0}, j}^{i}} v_{0}^{i-1}\left(l_{0}, \xi\right) d \xi, \quad \mathfrak{M}_{t_{0}, j}^{i}=M_{i-1}^{\ell_{0}} \cap K_{j}^{i}
$$

und

$$
G_{j}^{i}=\left\{(t, \xi) \in G^{i-1} \mid \text { es existiert ein } x \text { mit } \dot{x}(\tau)=w(\tau, x(\tau))\right.
$$

f. ü. auf $\left.\left(t_{0}, t_{1}\right), x(t)=\xi, x\left(t_{0}\right) \in \mathfrak{M}_{t_{0}, j}^{i}\right\}$

sei. Ist $\hat{\jmath} \in J$ durch

$$
\begin{aligned}
& \min _{j \in J^{\prime}}\left\{\int_{t_{0}}^{t_{1}} \int_{G} \int_{U} r(t, \xi, v) d \mu_{(t, \xi)}(v) v_{0, j}^{i}(t, \xi) d \xi d t\right. \\
& =\iint_{t_{0}} \int_{G} r(t, \xi, v) d \mu_{(t, \xi)}(v) v_{0, \hat{j}}^{i}(t, \xi) d \xi d t
\end{aligned}
$$

festgelegt, dann definieren wir

$$
v_{0}^{i}=v_{0 . \hat{\jmath}}^{i}, \quad \tilde{\mathfrak{w}}^{i}=v_{0}^{i}\left(\begin{array}{c}
1 \\
w
\end{array}\right), \quad \mathcal{M}_{t}^{i}=\mathfrak{M}_{t, \hat{\jmath}}^{i}, \quad G^{i}=G_{\hat{\jmath}}^{i}, \quad I^{i}=\dot{I}_{\hat{\imath}}^{i}
$$

Es gelten nun folgende Eigenschaften:

a) Für alle $i=0,1, \ldots$ ist

$$
\int_{\mathfrak{M}_{i}^{i}} v_{0}^{i}\left(t_{0}, \xi\right) d \xi=1
$$

Dies folgt mittels vollständiger Induktion, denn fïr $i=0$ gilt die Behauptung nach Voraussetzung, únd ist sie für $i-1$ richtig, so ist $v_{0}^{i}=v_{0, \hat{\jmath}}^{i}$ für ein $\hat{\jmath} \in J^{i}$ und folglich

sowie

$$
v_{0, \hat{\jmath}}^{i}(t, \xi)= \begin{cases}v_{0}^{i-1}\left(t_{0}, \xi\right) / L_{\hat{\jmath}}^{i} & \text { für } \xi \in \mathfrak{M}_{t_{0}, \hat{\jmath}}^{i} \\ 0 & \text { für } \xi \in \mathfrak{M}_{t_{0}} \backslash \mathfrak{M}_{t_{0}, \hat{\jmath}}^{i}\end{cases}
$$

$$
\iint_{\mathfrak{R}_{t_{0}}^{i}} v_{0}^{i}\left(t_{0}, \xi\right) d \xi=\left(1 / I^{i}\right) \int_{\mathfrak{M}_{t_{0}}^{i}} v_{0}^{i \cdot 1}\left(t_{0}, \xi\right) d \xi=1 .
$$

b) Es gilt

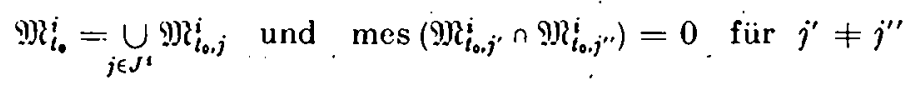

nach Definition und folglich auch $G^{i-1}=\cup\left\{G_{j}^{i}: j \in J^{i}\right\}$. Es ist dann

$$
\operatorname{mes}\left(G_{j^{\prime}}^{i} \cap G_{j^{\prime \prime}}^{i}\right)=0 \text { für alle } j^{\prime} \neq j^{\prime \prime} \text {, }
$$

denn wäré mes $\left(G_{j^{\prime}} \cap G_{j^{\prime \prime}}\right)>0$, dann betrachteten wir das Bild von $G_{j^{\prime}}^{i} \cap G_{j^{\prime}}^{i}$, bei der Abbildung

$$
\hat{\psi}:\left[i_{0}, t_{1}\right] \times\left(G_{j^{\prime}}^{i} \cap G_{j^{\prime \prime}}^{i}\right) \rightarrow \mathbf{R}^{n+1}, \quad \hat{\psi}(t, \xi) \doteq(t, \dot{\chi}(t, \xi))^{\mathbf{T}}
$$


mit der im Beweis des Hilfssatzes 1 definierten Funktion $\%$, und es gälte nach (15) wegen $|\partial \%(t, \xi) / \partial \xi|>0$ auch

$$
\operatorname{mes} \hat{\psi}\left(G_{j^{\prime}}^{i} \cap G_{j^{\prime \prime}}^{i}\right)=\int_{t_{0}}^{t_{1}} \int_{a_{j^{\prime}}^{i} \cap G_{j^{\prime \prime}}^{i}}\left|\frac{\partial \hat{\psi}(t, \xi)}{\partial(t, \xi)}\right| d \xi d t>0 .
$$

Andererseits ist aber für $j^{\prime} \neq j^{\prime \prime} \mathrm{im} \cdot$ Widerspruch dazu

$$
\operatorname{mes} \hat{\psi}\left(G_{j^{\prime}}^{i} \cap G_{j^{\prime \prime}}^{i}\right)=\operatorname{mes}\left(\left[t_{0}, \dot{t}_{1}\right] \times\left(\mathfrak{M} \mathbb{R}_{i_{0}, j^{\prime}}^{i} \cap \mathfrak{M R}_{t_{0, j},{ }^{\prime \prime}}^{i}\right)\right)=0 .:
$$

c) Aus, (16) und (17) ergibt sich weiter $v_{0, j}^{i-1}=\sum_{j \in J^{i}} I_{j}{ }^{i} v_{0, j}^{i}$.
d) Fiir $j \in J^{i}$ ist

$$
\begin{aligned}
. v_{0 . j}^{i}(t, \xi) & = \begin{cases}v_{0}^{i-1}(t, \xi) / I_{j}^{i}, & \text { für }(t, \xi) \in G_{i}{ }^{i} \\
0 & \text { für }(t, \xi) \in G^{0} \backslash G_{j}{ }^{i}\end{cases} \\
& = \begin{cases}v_{0}{ }^{0}(t, \xi) / I_{j}{ }^{i} I^{i-1} \ldots I^{1} & \text { für }(t, \xi) \in G_{j}^{i} \\
0 & \text { für }(t, \xi) \in G^{0} \backslash G_{j}{ }^{i} .\end{cases}
\end{aligned}
$$

Wir können Hilfssatz 1 anwendẹn, wobei $\tilde{v}_{0}=v_{0, j}^{i}, \tilde{I}=I_{j}^{i} I^{i-i} \ldots I^{1}$ und $\tilde{G}=G_{j}^{i}$ ist und erhalten.

$$
\int_{\mathfrak{M}_{t, j}^{i}} v_{0}^{0}(t, \xi) d \xi=I_{j}^{i} I^{i-1} \ldots I^{1}
$$

mit $\mathbb{M}_{t, j}^{i}=\mathrm{cl}\left\{\xi \in \bar{G} \mid v_{0, j}^{i}(t, \xi)>0\right\}$ bzw. für $t \in\left[t_{0}, t_{1}\right]$ und $i=0,1, \ldots$

$$
-\int_{\mathfrak{M}_{t, j}^{i} j} v_{0}^{i}(t, \xi) d \xi=1\left(j \in J^{i}\right), \text { speziell } \int_{\mathfrak{W}_{l}^{i}} v_{0}^{i}(t, \xi) d \xi=1 .
$$

e) Die Folge der Zielfunktionswerte

$$
\int_{t_{0}}^{t_{t}} \int_{G} \int_{U} \dot{r}(t, \xi, v) d \mu_{(t, \xi)}(v) v_{0}^{i}(t, \xi) d \xi d t
$$

zu $\tilde{\mathfrak{w}}^{i} \doteq v_{0}^{i}\left(\begin{array}{c}1 \\ w\end{array}\right)$ ist monoton fallend. $\mathrm{Da} v_{0}^{i-1}=\sum_{j \in J^{t}} I_{j}^{i} v_{0, j}^{i}$ und

$$
\sum_{j \in J^{t}} I_{j}^{i}=\sum_{j \in J^{\prime}} \int_{9 \mathbb{N}_{t_{0}, j}^{i}} v_{0}^{i-1}\left(t_{0}, \xi\right) d \xi=\int_{\mathbb{N}_{l_{0}}^{i-1}} v_{0}^{i-1}\left(t_{0}^{\prime}, \xi\right) d \xi=1
$$

ist; erhält man für $j=\hat{\jmath}$.

$$
\begin{aligned}
& \int_{t_{0}}^{t_{1}} \int_{G} \int_{U^{\prime}} r(t, \xi, v) d \mu_{(t, \xi)}(v) v_{0 . \hat{\jmath}}^{i}(t, \xi) d \xi d t \\
& \leqq \int_{t_{0}}^{t_{1}} \int_{G} \int_{U} r(t, \xi, v) d \mu_{(t, \xi)}(v) v_{0}^{t-1}(t, \xi) d \xi d t=\beta,
\end{aligned}
$$

denn anderénfalls wäre für alle $j \in J^{t}$

$$
\int_{l_{0}}^{t_{1}} \int_{G} \int_{U} r(t, \xi, v) d \mu_{(t, \xi)}(v) v_{0, j}^{i}(t, \xi) d \xi d t>\beta
$$


und somit

$$
\begin{aligned}
& \int_{t_{0}-G}^{t_{1}} \int_{U}-\int_{U} r(t, \xi, v) d \mu_{(t, \xi)}(v) v_{0}^{i-1}(t, \dot{\xi}) d \xi d t \\
& =\sum_{j \in J^{t}} I_{j}^{i} \int_{t_{0}}^{t_{1}} \int_{G} \int_{U} r(t, \xi, v) d \mu_{(t, \xi)}(v) v_{0, j}^{i}(t, \xi) d \xi d t>\beta .
\end{aligned}
$$

f) Schließlich gilt

$$
\lim _{n \rightarrow \infty} \int_{t_{0}}^{t_{2}} \int_{G} \int_{U} r(t, \xi, v) d \mu_{(t, \xi)}(v) v_{0}^{i}(t, \xi) d \xi d t=\int_{t_{0}}^{t_{1}} \int_{G} \int_{U} r(t, x(t), u(t)) d t
$$

für einen zulässigen ProzeB $(\stackrel{\prime}{x}, u) \in X_{0}^{\prime}$. Wir zeigen diese letzte Behauptung. Aus dem Konstruktionsprinzip ist klar, daß genau ein $\xi \in \cap 9\}_{t_{0}}^{i} \subset M \ell_{t_{0}}$ existiert. Folglich enthält $\cap G^{i}$ :genau ein $x$ mit

$$
\dot{x}(t)=w(t, x(t))=\int_{U} g(t, x(t), v) d \mu_{(t, x(t))}(v) \quad \text { f. ü. auf }{ }^{-}\left(t_{0}, t_{1}\right)^{\prime}
$$

und da $x$ Charakteristik von (10) und $v_{0}$ eindeutige Iösung des Anfangswertproblems $x\left(t_{0}\right) \in \mathfrak{M}_{l_{i}}$ zu (10) ist, folgt $x\left(t_{1}\right) \in \mathfrak{M}_{t_{1}}$. Es ist

$$
\lim _{i \rightarrow \infty} \int_{t_{0}}^{t_{1}} \int_{G} \int_{U} r(t, \xi, v) d \mu_{(t, \xi)}(v) v_{0}^{i}(t, \xi) d \xi d t=\lim _{i \rightarrow \infty} \int_{l_{0}}^{t_{1}} \int_{G} \bar{r}(t, \dot{\xi}) v_{0}^{i}(t, \xi) d \xi d t
$$

mit $\bar{r}(t, \xi)=\int_{U} r(t, \xi, v) d \mu_{(t, \xi)}(v)$ und wegen $\int_{\mathfrak{N} \mathbb{R}^{i}} v_{0}^{i}(t, \xi) d \xi=1$ folgt

$$
\begin{aligned}
& \lim _{i \rightarrow \infty} \int_{t_{0}}^{t_{1}} \int_{G} \bar{r}(t, \xi) v_{0}^{i}(t, \xi) d \xi d t=\lim _{i \rightarrow \infty} \int_{t_{0}}^{t_{1}} \int_{\mathfrak{N}_{t^{t}}} \bar{r}(t, \xi) v_{0}^{i}(t, \xi) d \xi d t \\
& \leqq \lim _{i \rightarrow \infty} \int_{t_{0}}^{t_{1}} \int_{\mathfrak{N}_{t^{t}}}\left[\sup _{\mathfrak{M}_{t^{t}}} \bar{r}(t, \xi)\right] v_{0}^{i}(t, \xi) d \xi d t \leqq \int_{t_{0}}^{t_{1}} \sup _{\mathfrak{M}_{t^{t}}} \bar{r}(t, \xi)^{-} d t .
\end{aligned}
$$

Wir verwenden einen Lebesgueschen Grenzwertsatz [1: Satz 13.8.1/S. 137] $:\left\{r_{i}\right\}$ mit $r_{i}(t) \doteq \sup \left\{r(t, \xi) \mid \xi \in \mathcal{M}_{t}{ }^{i}\right\}$ ist wegen $\mathfrak{M}_{t}{ }^{i} \subseteq \mathfrak{M}_{t}{ }^{i-1}$ monoton fallend, und wegen $x(t) \in \cap \mathfrak{M}_{t}{ }^{i}$ ist $r_{i}(\cdot) \geqq r(\cdot, x(t))$. für alle $i$. Also kann man den Grenzübergang $i \rightarrow \infty$ unter dem Integralzeichen ausführen und somit ist

$$
\lim _{i \rightarrow \infty} \int_{t_{0}}^{t_{1}} \sup _{\mathfrak{N}_{t^{t}}} \bar{r}(t, \xi) d t=\int_{t_{0}}^{t_{1}} \lim _{i \rightarrow \infty}\left(\sup _{\mathfrak{M}_{i}^{i}} \bar{r}(t, \xi)\right) d t \text {. }
$$
Wegen der Stetigkeit von $\dot{\bar{r}}$ in $x(t)$ nach Eigenschaft $(\mathrm{B})$ ist nun $\sup _{\mathfrak{M}_{t}^{t}} \bar{r}(t, \xi)=r(\ell, x(t))$
und folglich

$$
\int_{t_{0}}^{t_{1}} \lim _{i \rightarrow \infty}\left(\sup _{\mathfrak{M}_{t^{t}}} \bar{r}(t, \xi)\right) d t=\int_{t_{0}}^{t_{1}} \bar{r}(t, x(t)) d t=\int_{t_{0}}^{t_{1}} \int_{U} r(t, x(t), v) d \mu_{(t . x(t))}(v) d t
$$

bzw. zusammengefaßt

$$
\cdot \lim _{i \rightarrow \infty} \int_{i_{0}}^{t_{1}} \int_{G} \int_{U} r(t, \xi, v) d \mu_{(t, \xi)}(v) v_{0}^{i}(t, \xi) d \xi d t \leqq \int_{t_{0}}^{t_{1}} \int_{U} r(t, x(t), v) d \mu_{(t, x(t))}(v) d t .
$$


Analog zeigt man

und

$$
\lim _{i \rightarrow \infty} \int_{t_{0}}^{t_{i}} \int_{G} \int_{U} r(t, \xi, v) d \mu_{(t, \xi)} v_{0}^{i}(t, \xi) d \xi d t \geqq \lim _{i \rightarrow \infty} \int_{l_{0}}^{t_{1}} \inf _{\mathbb{R}_{t^{i}}}^{-} \bar{r}(t, \xi) d t
$$

$$
\lim _{i \rightarrow \infty} \int_{t_{0}}^{t_{1}} \inf \overline{D_{t^{\prime}}} \bar{r}(t, \xi) d t=\int_{t_{0}}^{t_{1}} \int_{0} r(t, x(t)) d \mu_{(t, x(t))}(v) d t .
$$

Also folgert man wiederum wegen der Stetigkeit von $\bar{r}$ in $x(t)$

$$
\lim _{i \rightarrow \infty} \int_{t_{0}}^{t_{1}} \int_{G} \int_{U} r(t, \xi, v) d \mu_{(t, \xi)}(v) \dot{v_{0}}(t, \ddot{\xi}) d \xi d t \geqq \int_{t_{0}}^{t_{1}} \int_{U} r_{1}(t, x(t)) d \mu_{(t, x(t))}(v) d t .
$$

Es ist $\mu^{*}=\left\{\mu_{(t, x(t))}=\mu_{t} \mid t \in\left[t_{0}, t_{1}\right]\right\}$ eine verallgemeinerte Steuerung zum Steuerproblem $\left(\mathbf{P}_{0}\right)$ im Sinne von L. C. Young [14: Kap. 3], zu der bei konvexem $U$ und . bei für alle $(t, \xi) \in\left[t_{0}, t_{1}\right] \times \bar{G}$ konvexen $r(t, \xi, \cdot)$ und $g_{i}(t, \xi, \cdot)(i=1, \ldots, n)$ eine zulässige Șteuerung $u$ zu $\left(\mathbf{P}_{0}\right)$ existiert, so daß nach (19) und (20) gilt

$$
\begin{aligned}
& \lim _{i \rightarrow \infty} \int_{t_{0}} \int_{G}^{t_{1}} \int_{U} r(t, \xi, v) d \mu_{(t, \xi)}(v) v_{0}^{i}(t, \xi) d \xi d t \\
& =\int_{t_{0}}^{t_{1}} \int_{U} r(t, x(t), v) d \mu(t, x(t))(v) d t=\int_{t_{0}}^{t_{1}} r(t, x(t), \stackrel{u}{u}(t)) d t ; \\
& \dot{x}(t)=g(t ; x(t), u(t)) \quad \text { f. ü. auf }\left(t_{0}, t_{1}\right), \quad x(t) \in \bar{G} ; \quad x\left(t_{0}\right) \in \mathfrak{M R _ { t _ { 0 } } , .} \\
& \dot{x}\left(t_{1}\right) \in \mathfrak{M} \ell_{t_{1}} .
\end{aligned}
$$

Mit anderen Worten, es ist $(x, u) \in X_{0}$, und nach Eigenschaft e) folgt die Behauptung des Satzes, $F^{\prime}(\mathfrak{w}) \geqq J(x, u)$.

Es ist zu vermuten, daß sich die Hauptideen des Satzes 3 auch auf mehrdimensionale Probleme übertragen lassen. Damit könnten auch in diesem Fall eine Beziehung zwischen dem Flußproblem (F) und dem Steuerproblem $\left(\mathbf{P}_{0}\right)$ hergestellt und Aussagen folgènder Art gewonnen werden: Liegt zwischen $\left(\mathbf{D}_{0}\right)$ und $(\mathbf{F})$ starke Dualität vor, so auch zwischen $\left(\mathbf{D}_{0}\right)$ und $\left(\mathbf{P}_{0}\right)$, wobei starke Dualitätsaussagen zwischen $\left(D_{0}\right)$ und $(F)$ einfacher zu gewinnen sind.

\section{LITERATUR}

[1] Dieddonxí, J.: Grundzüge der modernen Analysis, 'Bd. 2. Braunschweig: Friedr. Vieweg \& Sohn, und Berlin: Dt. Verlag Wiss. 1975.

[2] Dunford, N., and J. T. Schwarz: Linear Operators, Part I. New York: Intersc. Publ. 1958.

[3] Ekeland, I., and R. Temam: Convex Analysis and Variational Problems. Amsterdam: North:Holland Publ. Comp. and New York: Amer. Elsevier Publ. Comp., Inc. 1976.

[4] Göprfrt, A.: Mathematische Optimierung in allgemeinen Vektorräumen. Léipzig: BSB B. G. Teubner Verlagsges. 1973.

[5] Hildebrandt, S.: Uber die Identitït der Sobolewschen und Calkin-Morregschen Räume. Math. Annalen 148 (1962), 226-237.

[6] KaMKE, E: : Differentialgleichungen reeller Funktionen, Bd. 1. Leipzig: Akad. Verlagsges. Geest \& Portig K.-G. 1956.

[7] KLÖTZLER, R.: On a general conception of duality in optimal control. Lecture Notes in Math. 703 (1979), 189-196. 
[8] Krötzler, R.: Starke Dualität in der Steuerungstheorie. Math. Nachr. 95 (1977), 313 bis 320 .

[9] KíöTzLer, R.: Dualität bei Steuerungsproblemen und zugeordneten Flußproblemen I. Z. Anal. Anw. 1 (1982) 4, $47-57$.

[10] KLÖTZLER, R.:-Dualität bei Steuerungsproblemen und zugeordneten Flußproblemen II. Z. Anal. Anw. 2 (1983), 57-74.

[11] Pickenhain; S. ...Zur Dualität bei verallgemeinertén Steuerungsproblemẹn. Dissertation A. Leipzig: Karl-Marx-Úniversität 1983.

[12] Smirsow, W. I.: Lęhrgang der höheren Mathematik, Bd. 2. Berlin: Dt. Verlag Wiss. 1958.

[13] Vinter, R. B., and R. M. Lewis: Relaxation of Optimal Control Problems to Equivalent Convex Programs. J. Math. Anal. Appl. 74 (1983), 475-493.

[14] Young, L. C.: Lectures on the calculus of variations and optimal control theory. Philadelphia-London-Toronto: W. B. Saunders Comp. 1969. (

Manuskripteingang: 05. 09. 1984; in revidierter Fassung 25. 03.1986

\section{VERFASSER :}

Dr. Sabine Prckentiain

Sektion Mathematik der Karl-Marx-Universität

DRR - 7010 Leipzig, Karl-Marx-Platz 